\title{
A Method about Ebola Spread Forecasts Based on SIR
}

\author{
Han Dong \\ School of Computer Science, North China Electric Power University, Baoding, 071000, China. \\ 411135604@qq.com
}

Keywords: SIR model; Ebola.

\begin{abstract}
We predicts the future trends of Ebola. Following the SIR model, we construct the similar 'NSPC' model to describe the fluctuation of four classified cases. Because of the discreteness of data, we replace differential equations in the NSPC model with difference equations. Then according to historical data, we can adopt the nonlinear least squares method to obtain the spread equation and trend chart.
\end{abstract}

\section{The Classification of the Crowd}

In order to develop a reliable plan of drug manufacturing and delivery, we first need to learn the spread of Ebola virus in the future few month. Furthermore, we need to claim that our prediction model is established on the basis of existing data. Namely, we are in the absence of effective drug and vaccine to make predictions.

Obtaining the data from reliable resources (Wikipedia, the WHO's official website), we then divide the population into four categories according to the definition given by WHO.

We use conversion chart to represent those four groups as shown in figure 1.

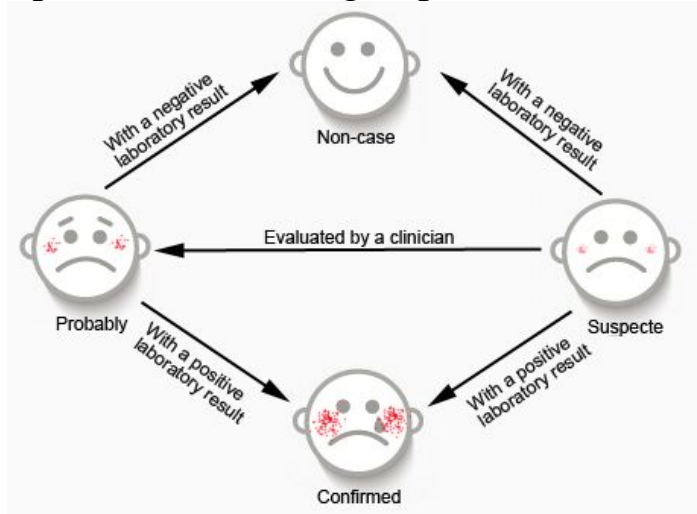

Figure 1. Conversion chart of four groups divided by WHO

NON-CASE: Any suspected or probable case with a negative laboratory result, "Non-case" showed no specific antibodies, RNA or specific detectable antigens.

SUSPECTED CASE: Any person, alive or dead, suffering or having suffered from a sudden onset of high fever and having had contact with:

- a suspected, probable or confirmed Ebola case;

- a dead or sick animal (for Ebola)

PROBABLE CASE: Any suspected case evaluated by a clinician.

CONFIRMED CASE: Any suspected or probably case with a positive laboratory result.

\section{The Establishment of Differential Equations}

\section{Ebola case contacts}

According to the WHO related documents, Ebola case contact can be defined as follow: Any person having been exposed to a suspect, probable or confirmed case of Ebola in at least one of the following ways:

- has slept in the same household with a case 
- has had direct physical contact with the case (alive or dead) during the illness

- has had direct physical contact with the (dead) case at the funeral

- has touched his/her blood or body fluids during the illness

- has touched his/her clothes or linens

- has been breastfed by the patient (baby)

To represent the decrease and increase number of the four groups, we aimed at these four groups to establish differential equation respectively. We first study the group classified as SUSPECTED CASE.

Under the condition of professional laboratory diagnosis, suspected case with a negative laboratory result will be transformed into the Non-case category every time period of Ebola virus transmission. Similarly, Any suspected or probably cases have the possibility to be diagnosed with a positive laboratory result. This kind of situation will lead to a decrease in suspected cases, while an increase in probable and confirmed cases. Besides, Depth and expand the check by medical personnel, new suspected cases will be found.

Based on the above analysis, we formulate the differential equation about suspected cases as follow:

$$
\frac{d S}{d t}=-K_{S N} S-K_{S P} S-K_{S C} S-K_{S} S+\lambda S+C T
$$

Where $C T$ denotes the new suspected cases of routine inspection, and $\lambda$ denotes the contact ratio with suspected cases.

In the same way, we can separately establish the differential equation about Non-case, Probable case and Confirmed case as follow:

$$
\begin{aligned}
& \frac{d N}{d t}=-K_{S N} S+K_{P N} P \\
& \frac{d P}{d t}=K_{S P} S-K_{P C} P-K_{P} P \\
& \frac{d C}{d t}=K_{S C} S+K_{P C} P-K_{C} C
\end{aligned}
$$

-For the suspected cases of people, considering they just have similar symptoms, people could still contact with them.

- For Probable cases and Confirmed cases, which are evaluated by clinic and have a positive laboratory result, they are in medical personnel monitoring. We assume they have no way contact with natural persons.

\section{Solution of the Differential Equation}

Step 1: To get the specific function express $S, N, P$ and $C$ on t respectively, we first simplify the differential equation by parameters Polymerization. Function (1) and (3) convert into the following expression (5) and (6):

$$
\begin{aligned}
& \frac{d S}{d t}=K_{S S} S+C T,\left(K_{S S}=-K_{S N}-K_{S P}-K_{S C}-K_{S}+\lambda\right) \\
& \frac{d P}{d t}=K_{P P} P+K_{S P} S,\left(K_{P P}=-K_{P C}-K_{P}\right)
\end{aligned}
$$

Step 2: Then transform the formula (5) (2) (6) (4) into the form of difference equations. With the use of least square method, we can determine the concrete numerical of parameters, such as $K_{s S}$, $K_{S N}, K_{P N}, K_{P P}, K_{S P}, K_{S C}, K_{P C}$ and $C T$.

Step 3: Finally we solve the first-order differential equations through nonlinear fitting method. With the help of MATLAB toolbox nilinfit function, we can get the fitting results.

Besides, we can receive the $\mathrm{S}, \mathrm{N}, \mathrm{P}, \mathrm{C}$ function expression on t (time) change. 


$$
\begin{aligned}
& S=a_{S} e^{K_{S S} t}+c_{S} \\
& N=a_{n 1} e^{K_{S S} t}+a_{n 2} e^{K_{P P} t}+c_{n} \\
& P=a_{p 1} e^{K_{S S} t}+a_{p 2} e^{K_{P P} t}+c_{p} \\
& C=a_{c 1} e^{K_{S 5} t}+a_{c 2} e^{K_{P P} t}+a_{c 3} e^{-K_{c} t}+C_{c}
\end{aligned}
$$

\section{Conclusion}

We counted 14 districts of Sierra Leone's population of infection and death toll separately, with time range from the August 2014 to February 2015. Larger amount of statistics help us get more accurate and realistic trend forecast. These 14 administrative regions are Bo, Bombali, Bonthe, kailahun, Kambia, Kenema, Koinadugu, Kono, Moyamba, port Loko, Pujehun, Tonkolili, Western Area urban, Western Area Rural.

As a result of the limitation of space, this paper only list the fitting results BO district as follow:

The value of parameter can be calculated by solving the differential equation according to the steps mentioned before. The results are shown in table 1.

Table 1 The value of parameter

\begin{tabular}{l|l|l}
\hline$K_{S S}=0.0156$ & $K_{S N}=0.5391$ & $K_{P N}=-0.3095$ \\
$K_{P P}=0.0203$ & $K_{S P}=0.0212$ & $K_{S C}=-0.02675$ \\
$K_{P C}=0.1663$ & $C T=3.6421$ & \\
\hline
\end{tabular}

Trend of changes in the number of

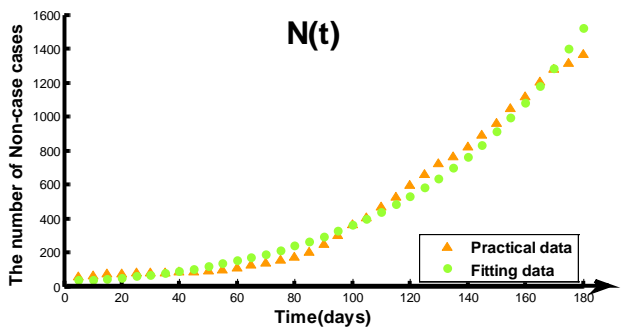

Figure 2. Non-case

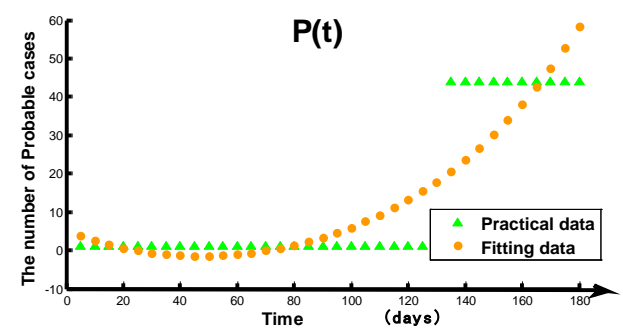

Figure 4. Probable case

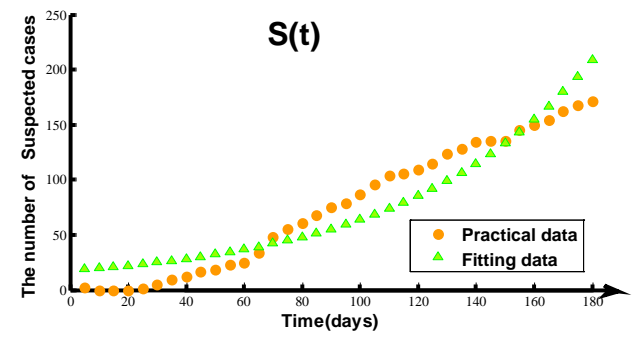

Figure 3. Suspected case

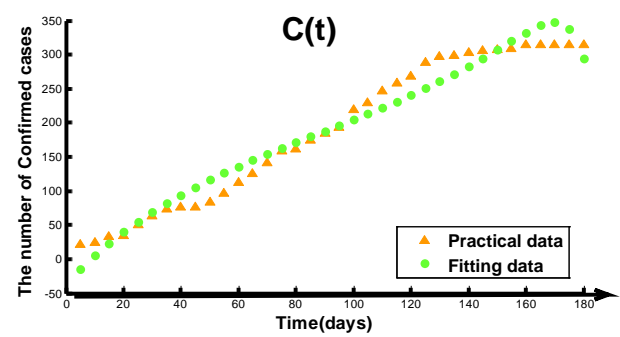

Figure 5. Ponfirmed case

By observing the number of population changes in the shape of the figures 2-5, we can get the following conclusion.

In the absence of effective drug and vaccine, the number of people in all four categories have been a surge in trend.

Ideal fitting results, verify the validity of our method.

At the same time, through nonlinear fitting, we can get the specific formula for number of changes about Non-case, Suspected case, Probable case and Confirmed case. The prediction equations are as follow:

$$
\begin{aligned}
& S=12.2967 e^{K_{S S} t}+5.6199 \quad N=98.5534 e^{K_{S S} t}+98.4208 e^{K_{P P} t}-114.7598 \\
& P=4.601 e^{K_{S S} t}+19.1881 e^{K_{P P} t}-18.4470 \quad C=15.2926 e^{K_{S S} t}-211.8422 e^{K_{P P} t}-160.02
\end{aligned}
$$

By expression of our income, we can separately predict the concrete number of Non-case, Suspected case, Probable case and Confirmed case in BO district of Sierra Leone, in a certain period 
of time in the next days. In the same we can calculate the prediction equation for the other 13 district of Sierra Leone.

\section{References}

[1] Paul Fine, Ken Eames, David L Heymann. "Herd Immunity": A Rough Guide J. Clinical Infectious Diseases. Oxford Unvi Press Inc, 2011(52): 911-916.

[2] http://www.who.int/csr/resources/publications/ebola/ebola-case-definition-contact-en.pdf 